\title{
Audiojogos educacionais: um Mapeamento Sistemático da Literatura
}

\author{
Walter R. B. Carvalho ${ }^{1}$, Carla L. Rodriguez ${ }^{1,2}$, Rafaela V. Rocha ${ }^{1,2}$ \\ ${ }^{1}$ Pós-graduação em Ciência da Computação, UFABC - Santo André, SP \\ ${ }^{2}$ Centro de Matemática, Computação e Cognição, UFABC - Santo André, SP \\ walter.carvalho@aluno.ufabc.edu.br, \{c.rodriguez, \\ rafaela.rocha\} eufabc.edu.br
}

\begin{abstract}
The number of researches on educational games in special education has been growing, and it is important to understand which technologies are relevant and how games can be used in pedagogical practice. This paper aims to present a systematic mapping of the literature on use and on development of audiogames in education. 11 papers were analyzed in order to identify the main technologies, disciplines and pedagogical theories addressed in these audiogames in the last 10 years. More than half of the audiogames used for educational purposes was applied in mathematics and languages, most of them also use speech synthesizers and screen readers. There was a lack of works that address pedagogical theories.
\end{abstract}

Resumo. Com a crescente necessidade de recursos voltados aos estudantes com deficiência visual, faltam estudos sobre o uso de audiojogos educacionais. Este artigo tem o objetivo de apresentar um mapeamento sistemático da literatura, sobre o uso e desenvolvimento de audiojogos aplicados na educação. Foram analisados 11 artigos, com o objetivo de identificar as principais tecnologias, conteúdos e teorias de aprendizagem abordadas em audiojogos educacionais nos últimos 10 anos. Mais da metade dos audiojogos usados para fins educacionais o aplicam em matemática e línguas, a maioria também utiliza sintetizadores de voz e leitores de tela. Foi observada uma carência de trabalhos que explicitam as teorias de aprendizagem adotadas.

\section{Introdução}

Nos últimos anos, houve um crescimento do percentual de estudantes com algum tipo de deficiência em escolas brasileiras, já que o número de matrículas na educação especial cresceu 34,7\% entre 2016 e 2020, alcançando 1,3 milhão de matrículas (INEP, 2021). Em 1996 foi instituída a Lei de Diretrizes e Bases da Educação Nacional, na qual regulamenta que deve ser assegurado o uso de metodologias adequadas e o atendimento especializado, com o objetivo de oferecer melhores condições de aprendizagem para estudantes com algum tipo de deficiência (Brasil, 1996), seguida pelas Diretrizes Nacionais para a Educação Especial na Educação Básica (Brasil, 2001). Existem diferentes tecnologias assistivas que possibilitam independência e autonomia para Pessoas com Deficiência $(\mathrm{PcD})$ visual no acesso a equipamentos digitais nos diferentes aspectos de sua vida, como no ambiente escolar, trabalho e lazer (Gasparetto et al., 2012). Entre estas ferramentas, podemos citar aplicativos de leitor de tela e sintetizador de voz, lupa, ajuste de tamanho e tipo de fonte, display e impressora Braille (Ferreira et al., 2008; Gasparetto et al., 2012).

A maioria dos jogos digitais tem a interface gráfica como a principal forma de interação do usuário com o jogo. Em grande parte, o som tem função de ambientação e 
imersão (Balan et al., 2015). Entretanto, alguns estudos analisaram o potencial de audiojogos no contexto educacional (Milne et al., 2014; Rovithis et al., 2014b; Hansen \& Hiraga, 2017), e construíram diretrizes para desenvolvimento de audiojogos (Rovithis et al., 2014a; Silva Ribeiro et al., 2019), bem como, aplicaram audiojogos em ambiente escolar e obtiveram aceitação por parte de educadores e estudantes com deficiência visual (Ferreira \& Cavaco, 2014; Araújo et al., 2016a; Sobral et al., 2017).

O desenvolvimento de jogos em sala de aula requer clareza sobre a abordagem curricular, a forma que ocorrerá a aprendizagem do estudante e qual método será recomendado para o educador alcançar os objetivos educacionais (Barbosa et al., 2008). Rocha et al. (2018) observam a falta de planejamento pedagógico durante o processo de desenvolvimento de ferramentas educacionais para o ensino de Libras (Língua brasileira de sinais), o que dificulta a sua aplicação pelos educadores em ambientes educacionais.

Nesse cenário, a presente pesquisa tem como objetivo oferecer uma visão geral do uso de audiojogos na educação por meio de um Mapeamento Sistemático da Literatura (MSL), com foco nos conteúdos trabalhados, nas abordagens educacionais, e nas tecnologias e características dos audiojogos. Este trabalho está organizado como a seguir: na Seção 2, são apresentados os trabalhos relacionados. Na Seção 3, é especificada a metodologia de MSL (etapas de planejamento, condução da pesquisa e análise de dados). Na Seção 4, são relatados e discutidos os resultados para cada questão de pesquisa. Por fim, na Seção 5, são descritas as considerações finais.

\section{Trabalhos Relacionados}

Silva Ribeiro et al. (2019) apresentaram resultados preliminares de uma Revisão Sistemática da Literatura (RSL) sobre audiojogos para PcD visual, com 13 artigos entre os anos de 2013 e 2018. O trabalho teve o objetivo de compreender quais as principais técnicas de som e formas de interação abordadas nesses jogos para acessibilidade. Os resultados apontam uma prevalência do uso de teclado em computadores, e o uso do touchscreen e sensor giroscópio para celular. Além disso, foi observado que essa categoria de jogo é capaz de oferecer uma sensação de imersão pela diversidade nas técnicas de som adotadas, criando sonoramente um ambiente virtual completo. Urbanek e Güldenpfennig (2019a) analisaram 48 audiojogos desenvolvidos nos últimos 20 anos, com base na metodologia da teoria fundamentada. Os principais pontos observados pelos autores são que: (1) os audiojogos, muitas vezes, são pensados para uso no ambiente acadêmico e não para o contexto de jogadores, para além da pesquisa; e (2) o processo de design de áudio vem sendo negligenciado. Garcez et al. (2020) fizeram uma RSL para investigar as melhores técnicas de desenvolvimento de jogos para PcD visual, com 17 artigos entre os anos 2004 e 2019. Os principais recursos de acessibilidade abordados nos artigos revisados foram o uso de efeitos sonoros e sonar. Em relação às temáticas, mais da metade dos jogos é voltado ao treinamento de navegação espacial, para a vida real, com uso de ambiente 3D.

Na RSL de Silva Ribeiro et al. (2019) apenas um audiojogo (Ferreira \& Cavaco, 2014) foi usado no contexto educacional, assim como em Urbanek e Güldenpfennig (2019a) (Kane et al., 2018). A RSL de Garcez et al. (2020) aborda jogos em geral para PcD visual e não apresentou audiojogos voltados à educação. Com base nesses estudos, o presente trabalho se aprofunda no estudo de audiojogos aplicados no contexto 
educacional, por meio de questões de pesquisa referentes às disciplinas e temas, contexto pedagógico (formal ou não-formal) e teorias de aprendizagem abordadas.

\section{Metodologia}

O Mapeamento Sistemático da Literatura (MSL) apresentado neste artigo foi conduzido com base no protocolo proposto por Kitchenham e Charters (2007) e uso da ferramenta Parsifal. Os passos realizados foram: (1) Planejamento: com definição das questões de pesquisa: busca e obtenção de trabalhos nas bases, seleção de estudos e extração de dados; e (3) Análise dos dados: descrição e discussão dos resultados.

\subsection{Planejamento}

A questão principal da pesquisa é "Qual é o estado da arte do uso de audiojogos na educação?". Busca-se um entendimento de quais conhecimentos e habilidades são explorados, e como são trabalhados em pesquisas voltadas ao uso de audiojogos na educação formal e não-formal, das características dos audiojogos e das tecnologias usadas no seu desenvolvimento. Dessa forma, foram definidas as seguintes questões de pesquisa: QP1: "Quais são os gêneros de jogos e tecnologias usadas no desenvolvimento de audiojogos educacionais?"; QP2: "Quais são as disciplinas e temas trabalhados em audiojogos e sua classificação quanto ao tipo de educação?"; e QP3: "Quais teorias pedagógicas são abordadas no uso de audiojogos educacionais?".

Para elaboração da string de busca, definiu-se inicialmente os termos principais "audiojogo" e "educação". Por ainda não haver um termo popularizado para audiojogos, diversos termos alternativos foram usados. A string usada foi: ("audiogame" OR "audio-based game" OR "audio game" OR "audio-only game" AND ("education" OR "classroom" OR "educational" OR "learning" OR "school"). A string em português não retornou artigos adicionais.

As bases de dados em inglês utilizadas para análise foram: ACM Digital Library, IEEE Digital Library, ISI Web of Science e Science@Direct. A base de dados em português utilizada foi a Comissão Especial em Informática na Educação (CEIE), na qual a string foi aplicada no Google Scholar junto com a sigla das conferências. Apesar da possibilidade de busca nas páginas de cada conferência, a string não alcançava palavras no conteúdo dos artigos. A forma que o Google Scholar faz a indexação de artigos permitiu a listagem de artigos relevantes para a pesquisa que não tinham os termos da string em seu título, resumo ou palavras-chave.

$\mathrm{Na}$ definição dos critérios de inclusão e exclusão (Quadro 1), definiu-se um recorte relacionado ao desenvolvimento ou uso de audiojogos em contexto educacional, seja ele formal ou não-formal; que tenha sido desenvolvido nos últimos 10 anos; que não seja um artigo duplicado, artigos publicados em mais de um periódico ou que fazem uso dos mesmos audiojogos em sala de aula; e também que não apresentem estudos secundários ou terciários, como no caso de revisões e mapeamentos.

\subsection{Condução da Pesquisa}

As buscas realizadas nas bases de dados retornaram 129 artigos científicos. Após a coleta, a primeira etapa foi a de exclusão de artigos duplicados, seguido do processo de 
exclusão de artigos com base nos critérios definidos. Após a verificação e leitura dos artigos na segunda etapa, oito artigos estavam adequados aos critérios de inclusão. Com o objetivo de encontrar outros artigos relevantes para o MSL, utilizou-se a metodologia de snowballing para frente e para trás, no qual foi possível encontrar mais três artigos. $\mathrm{O}$ método de snowballing para frente é definido como a análise das referências e citações dos estudos primários selecionados, enquanto o para trás refere-se aos trabalhos que citam os estudos primários (Wohlin, 2014), o que possibilita a adição de trabalhos relevantes. Dessa forma, o presente trabalho utiliza 11 artigos para responder às questões de pesquisa (conforme Tabela 1 com quantidade de artigos por base de dados).

Quadro 1. Critérios de exclusão e inclusão.

\begin{tabular}{|l|l|}
\hline \multicolumn{1}{|c|}{ Critérios de inclusão } & \multicolumn{1}{c|}{ Critérios de exclusão } \\
\hline $\begin{array}{l}\text { - Artigos que abordam o desenvolvimento ou uso } \\
\text { de audiojogos em contexto educacional }\end{array}$ & $\begin{array}{l}\text { - Artigos anteriores a } 2011 \\
\text { - Artigos duplicados } \\
\text { - Estudos secundários ou terciários }\end{array}$ \\
\hline
\end{tabular}

Tabela 1. Quantidade de artigos por base de dados

\begin{tabular}{|c|c|c|c|c|}
\hline Base de Dados & Importados & Selecionados & Snowballing & Total \\
\hline ACM Digital Library & 87 & 4 & 1 & 5 \\
\hline IEEE Digital Library & 7 & 0 & 1 & 1 \\
\hline ISI Web of Science & 11 & 1 & 0 & 1 \\
\hline Science@Direct & 20 & 0 & 0 & 0 \\
\hline CEIE/SBIE & 4 & 3 & 0 & 3 \\
\hline CEUR-WS.org & 0 & 0 & 1 & 1 \\
\hline Total & $\mathbf{1 2 9}$ & $\mathbf{8}$ & $\mathbf{3}$ & $\mathbf{1 1}$ \\
\hline
\end{tabular}

O Quadro 2 apresenta os artigos selecionados. Os artigos A1, A2 e A11 foram obtidos por meio da técnica de snowball. Dois deles (A1 e A2) têm origem nas bases de dados pesquisadas no projeto e não foram encontrados durante a condução da pesquisa com o uso da string. Isso ocorreu em A1 pois os autores categorizam seu artefato como um "game-based learning environment for visually impaired students", mas pode ser entendido como um audiojogo pelas suas características, como observado por Silva Ribeiro et al. (2019). No artigo A2, os autores citam audiojogos na pesquisa bibliográfica, e escolhem o uso do termo "braille-based game" para definir sua proposta, também pode ser categorizada como um audiojogo por ter uma interface de áudio para saída. A pesquisa foi feita para artigos publicados entre janeiro/2011 a março/2021, mas não foram encontradas publicações nos anos de 2011, 2012, 2013, 2015, 2019 e 2020. É possível observar que audiojogos como campo de pesquisa, e como comunidade indie de jogos acessíveis, ainda não tem termos popularizados e se encontram em construção. Além disso, todos os trabalhos são voltados ao uso de audiojogos no contexto da inclusão de PcD visual.

\subsection{Análise dos dados}

Os 11 audiojogos selecionados foram analisados de acordo com os aspectos: (1) Gênero do audiojogo (QP1): referente a diversidade da jogabilidade que um audiojogo pode ter (Kim et al., 2018); (2) Tecnologias utilizadas (QP1): referente aos tipos de dispositivos 
utilizados, além de hardware, software e complementos necessários para o funcionamento e acessibilidade dos audiojogos (Ferreira et al., 2008; Gasparetto et al., 2012); (3) Disciplina (QP2): referente à área do conhecimento e os temas trabalhados como objetivos de aprendizagem por meio do uso de audiojogos; (4) Tipo de Educação (QP2): baseado no espaço onde a educação foi trabalhada (Gohn, 2006); e (5) Teoria de aprendizagem (QP3): baseado na reprodução de modelos de processo de aprendizagem no qual o audiojogo atua dentro de uma prática pedagógica com base na classificação definida por Nagowah \& Nagowah (2009), e Ertmer \& Newby (2013). A planilha completa está disponível em: https://bit.ly/audiojogos-educacionais-msl.

Quadro 2. Artigos selecionados para o MSL.

\begin{tabular}{|c|c|c|c|c|c|c|}
\hline ID & Ano & Autores & $\begin{array}{l}\text { Base de } \\
\text { Dados }\end{array}$ & $\begin{array}{l}\text { Gênero de } \\
\text { Jogo }\end{array}$ & Disciplina & $\begin{array}{l}\text { Tipo de } \\
\text { educação }\end{array}$ \\
\hline $\mathrm{A} 1+$ & 2014 & $\begin{array}{c}\text { Ferreira \& } \\
\text { Cavaco }\end{array}$ & IEEE DL & Quiz & Matemática & Formal \\
\hline $\mathrm{A} 2+$ & 2014 & Milne et al. & ACM DL & Word Game & $\begin{array}{c}\text { Línguas/ } \\
\text { Braille }\end{array}$ & $\begin{array}{c}\text { Formal e } \\
\text { não-formal }\end{array}$ \\
\hline $\mathrm{A} 3^{*}$ & $2014 b$ & Rovithis et al. & ACM DL & RPG & Música & Formal \\
\hline A4 & $2016 a$ & Araújo et al. & ACM DL & $\begin{array}{c}\text { Arcade e Word } \\
\text { Game }\end{array}$ & $\begin{array}{c}\text { Línguas/ } \\
\text { Braille }\end{array}$ & Formal \\
\hline A5 & $2016 \mathrm{~b}$ & Araújo et al. & CEIE (SBIE) & Quiz & $\begin{array}{c}\text { Línguas/ } \\
\text { Braille }\end{array}$ & Formal \\
\hline A6 & 2017 & Hansen \& Hiraga & $\begin{array}{l}\text { Web of } \\
\text { Science }\end{array}$ & Puzzle & Música & $\begin{array}{l}\text { Formal e } \\
\text { não-formal }\end{array}$ \\
\hline A7 & 2017 & Sobral et al. & CEIE (SBIE) & RPG & \begin{tabular}{|c|}
$\begin{array}{c}\text { Matemática e } \\
\text { Línguas }\end{array}$ \\
\end{tabular} & Formal \\
\hline A8 & 2018 & Gerding et al. & CEIE (SBIE) & Puzzle & Geografia & Formal \\
\hline A9 & 2018 & Kane et al. & ACM DL & $\begin{array}{c}\text { Framework de } \\
\text { desenvolvimento }\end{array}$ & Programação & Formal \\
\hline $\mathrm{A} 10$ & 2018 & Marichal et al. & ACM DL & Puzzle & Matemática & Formal \\
\hline $\mathrm{A} 11^{*}+$ & 2018 & Rovithis \& Floros & CEUR-WS.org & RPG & Astronomia & Formal \\
\hline
\end{tabular}

Legenda: * audiojogos em desenvolvimento; + artigos adicionados manualmente

\section{Resultados e Discussões}

Nessa seção são reportados e discutidos os resultados do estudo e análise dos 11 artigos selecionados. O mapeamento foi conduzido no período de abril a junho/2021. As questões de pesquisa do MSL são respondidas a seguir.

\subsection{QP1: Quais são os gêneros de jogos e tecnologias usadas no desenvolvimento de audiojogos educacionais?}

Apenas em um artigo a aprendizagem ocorre enquanto os estudantes criam audiojogos (A9), os outros 10 artigos dedicam-se ao desenvolvimento ou uso de audiojogos. Um dos artigos (A4) faz uso de dois jogos em sala de aula, adaptações de asteroids (Arcade) e forca (Word Game) em audiojogos educacionais. Outros dois artigos (A3 e A11) não foram aplicados com voluntários pois os audiojogos (do gênero Role Playing Game RPG) ainda estavam sendo desenvolvidos. Do gênero de audiojogos aplicados: três 
artigos usam o gênero Puzzle (A6, A8 e A10), um faz uso de audiojogos de RPG (A7), dois abordam Quiz (A1 e A5), e um aplica audiojogo do gênero Word Game (A2).

Um dos aspectos mais importantes na implementação de audiojogos está relacionado ao uso de hardware e software capazes de oferecer acessibilidade ao usuário. Cinco trabalhos usaram audiojogos no computador (A1, A3, A7, A8 e A9), três no celular (A2, A4 e A5), dois no tablet (A6 e A10), e um não informa para qual tipo de dispositivo o jogo está sendo desenvolvido (A11). Em relação à interface de entrada, quatro usaram touchscreen do celular ou tablet (A2, A4, A5 e A6), dois usaram teclado (A3 e A8), dois também usaram mouse (A3 e A8), um usou display de Braille (sistema de escrita e leitura tátil) (A9), um blocos lógicos (brinquedo em madeira com diferentes peças) como interface tangível (A10), um joystick (A1), e dois não especificam o tipo de interface de entrada (A7 e A11). Em relação à interface de saída, audiojogos devem ter uma interface auditiva principal, podendo ou não ter uma interface gráfica complementar (Urbanek \& Güldenpfennig, 2019b). Dos trabalhos analisados, oito possuem algum tipo de efeito sonoro (A1, A3, A6, A7, A8, A9, A10 e A11), dois utilizam interface háptica (A2 e A10), dois utilizam vozes gravadas na narração (A1 e A8), e apenas dois não oferecem uma interface gráfica 2D complementar (A10 e A11). Em relação ao software e complementos necessários, seis usam narração por meio de sintetizador de voz ou leitor de tela (A2, A4, A5, A6, A7 e A9), três trabalhos usam teclado virtual em Braille (A2, A4 e A5), e um aborda ampliador de tela (A9). Os outros trabalhos (A1, A3, A8, A10 e A11) não informaram ou não usaram esses recursos.

\subsection{QP2: Quais são as disciplinas e temas trabalhados em audiojogos educacionais e sua classificação quanto ao tipo de educação?}

Os artigos estão contextualizados em espaço educacional, formal e não-formal, diretamente conectados às disciplinas e temas de seu currículo. Um deles trabalha de forma interdisciplinar (A7), nas matérias de línguas e matemática, mas não descreve os conteúdos abordados. Nos demais artigos, no total, seis disciplinas são abordadas: (1) Línguas: (3 artigos): um artigo (A5) aborda a prática do Braille para aprendizagem de ortografia, e dois (A2 e A4) a prática ou alfabetização do Braille por meio de exercícios de línguas; (2) Matemática (2 artigos): um (A1) aborda questões de probabilidade, proporcionalidade e funções, e um (A10) conceito de soma; (3) Música (2 artigos): abordam reconhecimento de timbre (A3 e A6), tom e ditado melódico (A3); (4) Astronomia (A11): aborda o conhecimento das camadas da atmosfera da Terra e os perigos do lixo espacial; (5) Programação (A9): aborda a aprendizagem da linguagem de programação JavaScript; e (6) Geografia (A8): aborda o conhecimento de países e suas capitais (A8).

Em relação ao tipo de educação na qual foi pensado o desenvolvimento ou uso de audiojogos, os 11 trabalhos são para a educação formal e dois deles citam, de forma explícita ou implícita, a aplicação na educação não-formal (A2 - uso com familiares - e A6 - para lazer). Seus propósitos são: (1) uso de audiojogos como suporte didáticometodológico para os professores em sala de aula (A1, A3, A4, A5, A7 e A11); (2) possibilidade da customização do jogo para objetivo educacional específico do professor em sala de aula (A5 e A8); (3) criação de audiojogos em grupo durante um 
workshop (A9); (4) treinamento (A6 - timbre e A10 - adição); e (5) possibilidade de uso acompanhado de pais e irmãos (A2).

\subsection{QP3: Quais teorias pedagógicas são abordadas no uso de audiojogos sérios?}

Apenas dois artigos nomeiam a teoria de aprendizagem utilizada: um artigo (A7) se baseia na teoria de aprendizagem Construtivista da linha piagetiana; e outro (A9) na abordagem Construcionista de Papert, teoria fundamentada no construtivismo (Amaral \& Sabotam, 2017). Isso indica que grande parte das pesquisas com audiojogos educacionais não aprofundam as abordagens pedagógicas adotadas para atingir seus objetivos educacionais. Entretanto, é possível compreender quais teorias são trabalhadas por meio da terminologia usada nos artigos para indicar o método de ensino, pressupostos de aprendizagem e forma de avaliação. Muitas vezes os educadores e desenvolvedores de recursos educacionais reproduzem as práticas de um movimento pedagógico baseados na própria experiência escolar, na técnica de colegas mais experientes ou no senso comum, sem necessariamente dominar a teoria (Libâneo, 1983).

Por meio da análise da terminologia, foi possível identificar trabalhos pautados na teoria de aprendizagem do Comportamentalismo (A1, A4 e A5), Cognitivismo (A3) e Construtivismo (A2 e A10, além de A7 e A9) . Não foi possível definir a teoria de aprendizagem utilizada em três artigos (A6, A8 e A11) pela ausência de termos que apontem a reprodução de uma determinada teoria de aprendizagem. Nas abordagens pedagógicas Comportamentalistas, foram encontrados termos como: enfoque na avaliação de desempenho do estudante (A1, A4 e A5), uso de sistema de recompensa para motivação (A1, A4 e A5), aprendizagem por meio da repetição de exercícios (A1, A4 e A5) e uso da Taxonomia dos Objetivos Educacionais (A4). Na abordagem da teoria de aprendizagem do Cognitivismo, encontramos elementos do jogo como analogia para o objetivo de aprendizagem e estruturação e sequenciamento das atividades para facilitar a aprendizagem (A3). Além do artigo baseado de forma explícita na teoria de aprendizagem do Construtivismo (A7) e a que cita o uso do Construcionismo (A9), outros dois artigos reproduzem as abordagens utilizadas pela pedagogia social e Construtivista de forma implícita (A2 e A10), e abordam: aprendizagem baseada em projetos (A7), aprendizagem colaborativa (A2 e A9), domínio do braille para domínio da leitura com objetivo de "aprender a aprender" (A2), construção de novas aprendizagens com conteúdos já conhecidos (A7), aprendizagem significativa (A7), aprendizagem ativa e lúdica (A10).

\section{Considerações finais}

Considerando o crescimento de matrículas de PcD visual nas escolas brasileiras, se faz necessário a elaboração de recursos didáticos acessíveis para a educação inclusiva. Neste cenário, a interface auditiva deve ser priorizada na elaboração de propostas pedagógicas acessíveis quando pensamos na implementação de jogos na educação, onde destaca-se o uso de audiojogos. O objetivo principal deste trabalho foi realizar um MSL para avaliar o estado da arte do uso e desenvolvimento de audiojogos na educação. A partir dos 11 artigos selecionados por meio dos critérios de inclusão e exclusão, foi possível responder às questões de pesquisas relacionadas às características tecnológicas e educacionais de audiojogos, bem como responder à questão principal da pesquisa "Qual é o estado da arte do uso de audiojogos na educação?". 
Em relação ao hardware utilizado, cinco trabalhos $(45,5 \%)$ usam computador, com o teclado e o mouse como principais dispositivos de entrada (18,2\% cada). Três trabalhos $(27,3 \%)$ são audiojogos para celular e dois $(18,2 \%)$ para tablet; dos quais o touchscreen é a interface de entrada mais usada (36,4\% do total). O uso de hardware como giroscópio e sonar, observado em audiojogos para treinamento, não possui exemplos de uso em audiojogos na educação, havendo potencial de ser utilizado como apoio na concretização de objetivos educacionais.

Destaca-se o uso de sintetizador de voz e leitores de tela (54,5\%), seguido de teclado virtual em braille $(27,3 \%)$ como software e complementos necessários implementados na interação dos usuários. Considerando a importância da interface auditiva, sintetizadores de voz e leitores de tela são capazes de substituir a função de um narrador por meio de uma tecnologia de conversão de texto em voz que lê todas as informações da tela. É possível afirmar que o destaque no uso de teclado virtual em braille decorre dos objetivos educacionais trabalhados nos audiojogos, já que a mesma quantidade de trabalhos $(27,3 \%)$ aborda a prática do braille na aprendizagem de línguas.

Os principais gêneros de jogos abordados são Puzzle e RPG (27,3\% cada). Além de um framework de desenvolvimento de jogos, os jogos se categorizam em apenas cinco gêneros, o mesmo pode ter relação com a dificuldade de se implementar jogos em contexto educacional com mecânicas voltadas à interface auditiva. A maioria dos audiojogos $(54,5 \%)$ focam no ensino de línguas e/ou matemática básica, seguido de dois artigos $(18,1 \%)$ voltados à educação musical. Esta quantidade tem relação com o potencial de implementação de teclado virtual em braille como interface para o usuário, e também pelo fato dos conteúdos abordados na educação musical serem predominantemente passíveis de aprendizagem com a interface auditiva.

Os 11 trabalhos foram aplicados ou sugeridos para uso dentro do contexto formal de educação, e entre eles, apenas dois trabalhos $(18,1 \%)$ exploraram também a educação não-formal. A maioria dos trabalhos (54,5\%) abordam audiojogos como ferramenta de suporte didático-metodológico para os professores em sala de aula, e dois possibilitam customização do jogo para objetivo educacional específico $(18,1 \%)$, viabilizando que professores trabalhem com outras disciplinas e conteúdos.

A principal teoria de aprendizagem usada é o construtivismo $(36,4 \%)$, seguido do comportamentalismo (27,3\%); mas apenas $18,1 \%$ especificam a teoria usada, mesmo com a importância em sua definição, já que a teoria de aprendizagem define a forma que o objetivo educacional deve ser atingido. A teoria construtivista segundo Nagowah e Nagowah (2009) pauta o conhecimento na aprendizagem ativa e na experiência gerada na interação do estudante com situação-problema, na qual é possível observar o potencial do uso de audiojogos na concretização de objetivos educacionais.

Como trabalhos futuros, sugere-se a elaboração de novos mapeamentos sistemáticos com outras abordagens de jogos educacionais como tecnologia assistiva para PcD visuais, por exemplo, jogos em braille. Outra proposta consiste na construção de audiojogos educacionais que contemplem explicitamente as abordagens pedagógicas, pelo potencial de favorecer a implementação de audiojogos como ferramenta didática em escolas que necessitam adotar práticas pedagógicas inclusivas. 


\section{Referências Bibliográficas}

Amaral, P. D. F., Sabota, B. (2017). Powtoon: análise do aplicativo web e seu potencial mediador na aprendizagem. Revista Tecnologia e Sociedade, 13(28), p. 72-89.

Araújo, M. C., Silva, A. R., Darin, T. G., Castro, E. L., Andrade, R. M., Lima, E. T., \& Viana, W. (2016a). Design and usability of a braille-based mobile audiogame environment. In: 31st Annual ACM Symposium on Applied Computing, p. 232-238.

Araújo, M. C., Silva, B. R., Queiroz, J. G., Viana, W. (2016b). Ortomonstro: um audiogame móvel customizável para práticas ortográficas de português por meio do braille. In: XXVII SBIE, p. 866-875.

Balan, O., Moldoveanu, A., Moldoveanu, F. (2015). Navigational audio games: an effective approach toward improving spatial contextual learning for blind people. International Journal on Disability and Human Development, 14(2), p. 109-118.

Barbosa, R. C., Barbosa, R. C., Tavares, R., dos Santos, J. N., Rodrigues, G. L., Andrade, M. (2008) O Jogo Educacional como Recurso Digital e a Aprendizagem Significativa de Gramática. In: XIX SBIE, p. 491-500.

Brasil, L. (1996). Lei 9.394, de 20 de dezembro de 1996. Estabelece as Diretrizes e Bases da Educação Nacional. Diário Oficial da União.

Brasil. Ministério da Educação (2001). Resolução CNE/CEB No 2, de 11 de Fevereiro de 2001. Diretrizes nacionais para a educação especial na educação básica. Brasília: Secretaria da Educação Especial.

Ertmer, P. A., Newby, T. J. (1993). Behaviorism, cognitivism, constructivism: Comparing critical features from an instructional design perspective. Performance improvement quarterly, 6(4), p. 50-72.

Ferreira, F., Cavaco, S. (2014). Mathematics for all: a game-based learning environment for visually impaired students. In: IEEE Frontiers in Education Conference, p. 1-8.

Ferreira, S. B. L., Rodrigues, R. N. (2008). e-Usabilidade. Grupo Gen-LTC.

Garcez, L., Thiry, M., Fernandes, A. (2020). Accessible Features to Support Visually Impaired People in Game Development: a systematic literature review of the last 15 years, In: 15th Iberian Conference on Information Systems and Technologies, p. 1-6.

Gasparetto, M. E. R. F., Montilha, R. D. C. I., de Paula Arruda, S. M. C., Sperque, J., de Azevedo, T. L., Souza Nobre, M. I. R. (2012). Utilização de recursos de tecnologia assistiva por escolares com deficiência visual. Informática na educação: teoria \& prática, 15(2), p. 113-130.

Gerding, T. M., Otsuka, J., Junior, P. H. F. (2018). Design and development of a memory game solution for players with and without visual impairment. In: XXIX SBIE, p. 785-794.

Gohn, M. G. (2006). Educação não-formal, participação da sociedade civil e estruturas colegiadas nas escolas. Revista Ensaio-Avaliação e Políticas Públicas em Educação, 14(50), p. 11-25.

Hansen, K. F., Hiraga, R. (2017). The effects of musical experience and hearing loss on solving an audio-based gaming task. Applied Sciences, 7(12), 1278, p. 1-19.

INEP. (2021) “Censo da Educação Básica 2020: notas estatísticas”. Disponível em: https://download.inep.gov.br/. Acesso: jun/2021. 
Kane, S. K., Koushik, V., Muehlbradt, A. (2018). Bonk: accessible programming for accessible audio games. In: 17th ACM Conference on Interaction Design and Children, p. 132-142.

Kim, S., Song, K., Lockee, B., \& Burton, J. (2018). What Is a Game?. Gamification in Learning and Education, p. 15-23.

Libâneo, J. C. (1983). Tendências pedagógicas na prática escolar. Revista da Associação Nacional de Educação, 3, p. 11-19.

Marichal, S., Rosales, A., Sansone, G., Pires, A. C., Bakala, E., Perilli, F. G., Blat, J. (2018). LETSmath. In: 20th International Conference on Human-Computer Interaction with Mobile Devices and Services Adjunct, p. 313-320.

Milne, L. R., Bennett, C. L., Ladner, R. E., \& Azenkot, S. (2014). BraillePlay: educational smartphone games for blind children. In: 16th International ACM SIGACCESS Conference on Computers \& Accessibility, p. 137-144.

Nagowah, L., Nagowah, S. (2009). A Reflection on the Dominant Learning Theories: Behaviourism, Cognitivism and Constructivism. International Journal of Learning, 16(2), p. 279-285.

Rocha, P. S. R., de Lima, R. W., Queiroz, P. G. G. (2018). Tecnologias para o ensino da Língua Brasileira de Sinais (LIBRAS): Uma revisão sistemática da literatura. Revista Brasileira de Informática na Educação, 26(03), 42.

Rovithis, E., Floros, A., Mniestris, A., Grigoriou, N. (2014a). Audio games as educational tools: Design principles and examples. In: 2014 IEEE Games Media Entertainment, p. 1-8.

Rovithis, E., Mniestris, A., Floros, A. (2014b). Educational audio game design: sonification of the curriculum through a role-playing scenario in the audio game 'Kronos'. In: 9th International Audio Mostly Conference, p. 1-6.

Rovithis, E., Floros, A. (2018). AstroSonic: an educational audio gamification approach. In: DCAC Conference, Interdisciplinary Creativity in Arts and Technology, p. 116-123.

Silva Ribeiro, D., Silva, I. R., Lobo, T., Borges, L., Nunes, E. (2019). Uma Revisão Sistemática Sobre Técnicas de Audio Games Para Promover Acessibilidade de Pessoas com Deficiência Visual no Uso de Games. In: X ERI-MT, p. 103-108.

Sobral, F., Umeres, L., Schanoski, W., Bartelmebs, R., Assis, M. (2017). A Utilização de Role Playing Games Digitais como Ferramenta Complementar no Processo de Aprendizagem de Crianças Deficientes Visuais. In: XXVIII SBIE, p. 635-644.

Urbanek, M., Güldenpfennig, F. (2019a). Celebrating 20 years of computer-based audio gaming. In: 14th International Audio Mostly Conference, p. 90-97.

Urbanek, M., Güldenpfennig, F. (2019b). Unpacking the audio game experience: Lessons learned from game veterans. In Proceedings of the Annual Symposium on Computer-Human Interaction in Play, p. 253-264.

Wohlin, C. (2014). Guidelines for snowballing in systematic literature studies and a replication in software engineering. In: 18th international conference on evaluation and assessment in software engineering, p. 1-10. 